\title{
Relation of the oscillator and Coulomb systems on spheres and pseudospheres
}

\author{
Armen Nersessian ${ }^{1,2}$, George Pogosyan ${ }^{1,2}$ \\ ${ }^{1}$ Bogoliubov Laboratory of Theoretical Physics, Joint Institute for Nuclear Research, 141980 Dubna, Russia \\ ${ }^{2}$ Department of Theoretical Physics and International Center for Advanced Studies, \\ Yerevan State University, A. Manoogian St., 1, Yerevan, 375025 Armenia
}

(October 28, 2018)

\begin{abstract}
It is shown, that oscillators on the sphere and the pseudosphere are related, by the so-called Bohlin transformation, with the Coulomb systems on the pseudosphere. The even states of an oscillator yield the conventional Coulomb system on the pseudosphere, while the odd states yield the Coulomb system on the pseudosphere in the presence of magnetic flux tube generating spin $1 / 2$. A similar relation is established for the oscillator on the (pseudo)sphere specified by the presence of constant uniform magnetic field $B_{0}$ and the Coulomb-like system on pseudosphere specified by the presence of the magnetic field $\frac{B}{2 r_{0}}\left(\left|\frac{x_{3}}{\mathbf{x}}\right|-\epsilon\right)$. The correspondence between the oscillator and the Coulomb systems the higher dimensions is also discussed.
\end{abstract}

PACS number(s)03.65-w

The ( $d$-dimensional) oscillator and Coulomb systems are the most known representatives of mechanical systems with hidden symmetries which define the $s u(d)$ symmetry algebra for the oscillator, and $s o(d+1)$ for the Coulomb system. The hidden symmetry has a very transparent meaning in the case of oscillator while in the case of the Coulomb system it has a more complicated interpretation in terms of geodesic flows of a $d$-dimensional sphere [1]. On the other hand, both in classical and quantum cases, the transformation $r=R^{2}$ converts the $(p+1)$-dimensional radial Coulomb problem to a $2 p$-dimensional radial oscillator ( $r$ and $R$ denote the radial coordinates of Coulomb and oscillator systems, respectively). In three distinguished cases, $p=1,2,4$, one can establish a complete correspondence between the Coulomb and the oscillator systems, by making use of the so-called Bohlin (or Levi-Civita) [2], KustaanheimoStiefel [3] and Hurwitz [4] transformations, respectively (see also [5]). These transformations imply the reduction of the oscillator system by an action of $Z_{2}, U(1), S U(2)$ group, respectively, and yield the Coulomb-like systems specified by the presence of monopoles 6 , 8 .

On the other hand, the oscillator and Coulomb systems admit the generalizations to a $d$-dimensional sphere and a two-sheet hyperboloid (pseudosphere) with a radius $R_{0}$ given by the potentials [9, 10 ]

$$
V_{o s c}=\frac{\alpha^{2} R_{0}^{2}}{2} \frac{\mathbf{x}^{2}}{x_{d+1}^{2}}, \quad V_{C}=-\frac{\gamma}{R_{0}} \frac{x_{d+1}}{|\mathbf{x}|},
$$

where $\mathbf{x}, x_{d+1}$ are the (pseudo)Euclidean coordinates of the ambient space $\mathbb{R}^{\mathrm{d}+1}\left(\mathbb{R}^{\mathrm{d} .1}\right): \epsilon \mathbf{x}^{2}+x_{d+1}^{2}=R_{0}^{2}, \epsilon= \pm 1$. The $\epsilon=+1$ corresponds to the sphere and $\epsilon=-1$ corresponds to the pseudosphere. These systems possess nonlinear hidden symmetries providing them with the properties similar to those of conventional oscillator and Coulomb systems. Notice that the oscillators on sphere and pseudosphere have isomorphic configuration spaces ( $d$-dimensional plane with a cut circle, in the stereographic projection), since the first one is undefined on the equator $x_{d+1}=0$. The Coulomb system is attractive/repulsive on the upper/lower hemisphere and has the same behavior on both the sheets of the hyperboloid. These systems have been investigated by various methods from many viewpoints (see, e. g. 11 and refs therein).

How to relate the oscillator and Coulomb systems on (pseudo)spheres?

This question seems to be crucial for understanding the geometrical meaning of the hidden symmetries of Coulomb systems on (pseudo)spheres and for the construction of their generalizations, as well as for the twistor description of the relativistic spinning particles on AdS spaces. In Ref. [12] devoted to this problem, the oscillator and Coulomb systems on spheres were related by mappings containing transitions to imaginary coordinates.

In the present letter, we establish the transparent correspondence between oscillator and Coulomb systems on (pseudo)spheres for the simplest, two-dimensional, case $(p=1)$ that can be extended easily to higher dimensions $(p=2,4)$. We show that, under stereographic projection the conventional Bohlin transformation relates the two-dimensional oscillator on the (pseudo)sphere to the Coulomb system on pseudosphere, as well as those interacting with specific external magnetic fields. This simple construction allows immediately to connect the generators of the hidden symmetries of the systems under consideration, as well as to clarify the mappings suggested in 12 .

Let us introduce the complex coordinate $z$ parametrizing the sphere by the complex projective plane $\mathbb{C P}^{1}$ and the two-sheeted hyperboloid by the Poincaré disks $\mathcal{L}$

$$
\mathbf{x} \equiv x_{1}+i x_{2}=R_{0} \frac{2 z}{1+\epsilon z \bar{z}}, \quad x_{3}=R_{0} \frac{1-\epsilon z \bar{z}}{1+\epsilon z \bar{z}} .
$$

so that the metric becomes conformally-flat

$$
d s^{2}=R_{0}^{2} \frac{4 d z d \bar{z}}{(1+\epsilon z \bar{z})^{2}}
$$


The lower hemisphere and the lower sheet of the hyperboloid are parametrized by the unit disk $|z|<1$, while the upper hemisphere and the upper sheet of hyperboloid are specified by $|z|>1$, and transform one into another by the inversion $z \rightarrow 1 / z$. Since in the limit $R_{0} \rightarrow \infty$ the lower hemisphere (the lower sheet of hyperboloid) converts into the whole two-dimensional plane, we have to restrict ourselves by those defined on the lower hemisphere and the lower sheet of hyperboloid (pseudosphere), to keep the correspondence with conventional oscillator and Coulomb systems. In these terms the oscillator and Coulomb potentials read

$$
V_{o s c}=\frac{2 \alpha^{2} R_{0}^{2} z \bar{z}}{(1-\epsilon z \bar{z})^{2}}, \quad V_{C}=-\frac{\gamma}{R_{0}} \frac{1-\epsilon z \bar{z}}{2|z|},
$$

Notice that the parametrization (2) is nothing but the stereographic projection of the two-dimensional (pseudo)sphere

$$
\frac{z}{2 R_{0}}=\left\{\begin{array}{cc}
\cot \frac{\theta}{2} \mathrm{e}^{i \varphi} & \text { for sphere } \\
\operatorname{coth} \frac{\theta}{2} \mathrm{e}^{i \varphi} & \text { for pseudosphere }
\end{array}\right.
$$

where $\theta, \varphi$ are the (pseudo)spherical coordinates.

Let us equip the oscillator's phase space $T^{*} \mathbb{C P}^{1}\left(T^{*} \mathcal{L}\right)$ with the symplectic structure

$$
\omega=d \pi \wedge d z+d \bar{\pi} \wedge d \bar{z}
$$

and introduce the rotation generators defining $s u(2)$ algebra if $\epsilon=1$ and $s u(1.1)$ algebra if $\epsilon=-1$

$$
\mathbf{J} \equiv \frac{i J_{1}-J_{2}}{2}=\pi+\epsilon \bar{z}^{2} \bar{\pi}, \quad J \equiv \frac{\epsilon J_{3}}{2}=i(z \pi-\bar{z} \bar{\pi}) .
$$

These generators, together with $\mathbf{x} / R_{0}, x_{3} / R_{0}$ define the algebra of motion of the (pseudo)sphere via the following nonvanishing Poisson brackets

$$
\begin{gathered}
\{\mathbf{J}, \mathbf{x}\}=2 x_{3},\left\{\mathbf{J}, x_{3}\right\}=-\epsilon \overline{\mathbf{x}},\{J, \mathbf{x}\}=i \mathbf{x}, \\
\{\mathbf{J}, \overline{\mathbf{J}}\}=-2 i \epsilon J,\{\mathbf{J}, J\}=i \mathbf{J} .
\end{gathered}
$$

In these terms, the Hamiltonian of free particle on the (pseudo)sphere reads

$$
H_{0}^{\epsilon}=\frac{\mathbf{J} \overline{\mathbf{J}}+\epsilon J^{2}}{2 R_{0}^{2}}=\frac{(1+\epsilon z \bar{z})^{2} \pi \bar{\pi}}{2 R_{0}^{2}}
$$

while the oscillator's Hamiltonian is given by the expression

$$
H_{o s c}^{\epsilon}\left(\alpha, R_{0} \mid \pi, \bar{\pi}, z, \bar{z}\right)=\frac{(1+\epsilon z \bar{z})^{2} \pi \bar{\pi}}{2 R_{0}^{2}}+\frac{2 \alpha^{2} R_{0}^{2} z \bar{z}}{(1-\epsilon z \bar{z})^{2}} .
$$

It can be easily verified, by the use of (8), that the latter system possesses the hidden symmetry given by the complex (or vectorial) constant of motion 10

$$
\mathbf{I}=I_{1}+i I_{2}=\frac{\mathbf{J}^{2}}{2 R_{0}^{2}}+\frac{\alpha^{2} R_{0}^{2}}{2} \frac{\overline{\mathbf{x}}^{2}}{x_{3}^{2}}
$$

which defines, together with $J$ and $H_{o s c}$, the cubic algebra

$$
\{\mathbf{I}, J\}=2 i \mathbf{I}, \quad\{\overline{\mathbf{I}}, \mathbf{I}\}=4 i\left(\alpha^{2} J+\frac{\epsilon J H_{o s c}}{R_{0}^{2}}-\frac{J^{3}}{2 R_{0}^{4}}\right) .
$$

The energy surface of the oscillator on the (pseudo)sphere $H_{\text {osc }}^{\epsilon}=E$ reads

$$
\frac{\left(1-(z \bar{z})^{2}\right)^{2} \pi \bar{\pi}}{2 R_{0}^{4}}+2\left(\alpha^{2}+\epsilon \frac{E}{R_{0}^{2}}\right) z \bar{z}=\frac{E}{R_{0}^{2}}\left(1+(z \bar{z})^{2}\right) .
$$

Now, performing the canonical Bohlin transformation [2]

$$
w=z^{2}, \quad p=\frac{\pi}{2 z}
$$

one can rewrite the expression (13) as follows:

$$
\frac{(1-w \bar{w})^{2} p \bar{p}}{2 r_{0}^{2}}-\frac{\gamma}{r_{0}} \frac{1+w \bar{w}}{2|w|}=\mathcal{E}_{C},
$$

where we introduced the notation

$$
r_{0}=R_{0}^{2}, \quad \gamma=\frac{E}{2}, \quad-2 \mathcal{E}_{C}=\alpha^{2}+\epsilon \frac{E}{r_{0}} .
$$

Comparing the l.h.s. of (15) with the (4), (9) we conclude that (15) defines the energy surface of the Coulomb system on the pseudosphere with "radius" $r_{0}$, where $w, p$ denote complex stereographic coordinate and its conjugated momentum, respectively. $r_{0}$ is the "radius" of pseudosphere, while $\mathcal{E}_{C}$ is the system's energy.

Hence, the Bohlin transformation of the classical isotropic oscillator on the (pseudo)sphere yields the classical Coulomb problem on the pseudosphere.

The constants of motion of the oscillators, $J$ and $\mathbf{I}$ (which coincide on the energy surfaces (13)) are converted, respectively, into the doubled angular momentum and the doubled Runge-Lenz vector of the Coulomb system

$$
J \rightarrow 2 J_{C}, \quad \mathbf{I} \rightarrow 2 \mathbf{A}, \quad \mathbf{A}=-\frac{i J_{C} \mathbf{J}_{C}}{r_{0}}+\gamma \frac{\overline{\mathbf{x}}_{C}}{\left|\mathbf{x}_{C}\right|},
$$

where $\mathbf{J}_{C}, J_{C}, \mathbf{x}_{C}$ denote the rotation generators and the pseudo-Euclidean coordinates of the Coulomb system.

The quantum-mechanical counterpart of the energy surface 13 is the Schrödinger equation

$$
\mathcal{H}_{\text {osc }}^{\epsilon}\left(\alpha, R_{0} \mid \pi, \bar{\pi}, z, \bar{z}\right) \Psi(z, \bar{z})=E \Psi(z, \bar{z}),
$$

with the quantum Hamiltonian defined (due to the twodimensional origin of the system) by the expression (10), where $\pi, \bar{\pi}$ are the momenta operators (hereafter we assume $\hbar=1$ )

$$
\pi=-i \frac{\partial}{\partial z}, \quad \bar{\pi}=-i \frac{\partial}{\partial \bar{z}}
$$


The energy spectrum of this system is given by the expression (see e.g. 11] and refs therein)

$$
E=\tilde{\alpha}(N+1)+\epsilon \frac{(N+1)^{2}}{2 R_{0}^{2}}, \quad N=2 n_{r}+|M|,
$$

where $\tilde{\alpha}=\sqrt{\alpha^{2}+1 /\left(4 R_{0}^{4}\right)}, M$ is the eigenvalue of $J, N$ is the principal quantum number, $n_{r}$ is the radial quantum number,

$$
\begin{gathered}
|M|=0,1, \ldots, N, n_{r}=0,1, \ldots[N / 2], \\
N=0,1, \ldots, N_{\text {max }}=\left\{\begin{array}{cc}
\infty, & \text { if } \epsilon=1 \\
{\left[2 \tilde{\alpha} R_{0}^{2}\right]-1,} & \text { if } \epsilon=-1
\end{array}\right.
\end{gathered}
$$

So, the the number of levels in the energy spectrum of the oscillator is infinite on the sphere and finite on the pseudosphere. The degeneracy of the energy spectrum is the same as in the flat case, viz $2 N+1$.

The quantum-mechanical correspondence between oscillator and Coulomb systems is more complicated, because the Bohlin transformation (14) maps the $z$-plane in the two-sheeted Riemann surface, since arg $w \in[0,4 \pi)$. Thus, we have to supplement the quantum-mechanical Bohlin transformation with the reduction by the $Z_{2}$ group action, choosing either even $(\sigma=0)$ or odd $(\sigma=1 / 2)$ wave functions

$$
\begin{gathered}
\Psi_{\sigma}(z, \bar{z})=\psi_{\sigma}\left(z^{2}, \bar{z}^{2}\right)(z / \bar{z})^{2 \sigma}: \\
\psi_{\sigma}(|w|, \arg w+2 \pi)=\psi_{\sigma}(|w|, \arg w) .
\end{gathered}
$$

This implies that the range of definition of $w$ can be restricted, without loss of generality, to $\arg w \in[0,2 \pi)$. In that case, the resulting system is the Coulomb problem on the hyperboloid given by the Schrödinger equation

$$
H_{C}^{-}\left(\gamma, r_{0} \mid p_{\sigma}, \bar{p}_{\sigma}, w, \bar{w}\right) \psi_{\sigma}=\mathcal{E}_{C} \psi_{\sigma}
$$

where $\gamma, \mathcal{E}_{C}, r$ are given by $16, H_{C}^{-}$denotes the Hamiltonian of the Coulomb system on pseudosphere with the momenta operators

$$
p_{\sigma}=-i \frac{\partial}{\partial w}-\frac{\sigma}{i w}, \quad \bar{p}_{\sigma}=-i \frac{\partial}{\partial \bar{w}}+\frac{\sigma}{i \bar{w}} .
$$

Hence, the resulting Coulomb system includes the interaction with the magnetic vortex (an infinitely thin solenoid) with the magnetic flux $\pi \sigma$ and zero strength rot $\sigma / w=0$. Such composites are tipical representatives of anyonic systems with the spin $\sigma$ [13]. So, we get a conventional $2 d$ Coulomb problem on the hyperboloid at $\sigma=0$ and those with spin $1 / 2$ generated by the magnetic flux, at $\sigma=1 / 2$. Taking into account the relations (16), one can rewrite the oscillator's energy spectrum (20) as follows

$$
\sqrt{\frac{1}{4 r_{0}^{2}}-\epsilon \frac{2 \gamma}{r_{0}}-2 \mathcal{E}_{C}}=\frac{2 \gamma}{N+1}-\epsilon \frac{N+1}{2 r_{0}} .
$$

From this expression one can easily obtain the energy spectrum of the reduced system on the pseudosphere

$$
\mathcal{E}_{C}=-\frac{N_{\sigma}\left(N_{\sigma}+1\right)}{2 r_{0}^{2}}-\frac{\gamma^{2}}{2\left(N_{\sigma}+1 / 2\right)^{2}},
$$

where

$$
\begin{gathered}
N_{\sigma}=n_{r}+m_{\sigma}, \quad m_{\sigma}=M / 2, \\
n_{r}, m_{\sigma}-\sigma, N_{\sigma}-\sigma=0,1, \ldots, N_{\sigma}^{\max }-\sigma .
\end{gathered}
$$

Here $m_{\sigma}$ denotes the eigenvalue of the angular momentum of the reduced system, and $n_{r}$ is the radial quantum number of the initial (and reduced) system. Notice, that the magnetic vortex shifts the energy levels of the twodimensional Coulomb system which is nothing but the reflection of Aharonov-Bohm effect.

It is seen, that the whole spectrum of the oscillator on pseudosphere $(\epsilon=-1)$ transforms into the spectra of the constructed Coulomb systems on the pseudosphere, while for the oscillator on the sphere $(\epsilon=1)$ the positivity of l. h. s. of (25) restricts the admissible values of $N_{\sigma}$. So, only the part of the spectrum of the oscillator on the sphere transforms into the spectrum of Coulomb system. Hence, in both cases we get the same result

$$
N_{\sigma}^{\max }=\left[\sqrt{r_{0} \gamma}-(1 / 2+\sigma)\right] .
$$

To obtain the flat limit we perform the rescaling

$$
(z, \pi) \rightarrow\left(\frac{z}{2 R_{0}}, 2 R_{0} \pi\right), \quad(w, p) \rightarrow\left(\frac{w}{4 r_{0}}, 4 r_{0} p\right),
$$

where $r_{0}=R_{0}^{2}$, and then take the limit $R_{0} \rightarrow \infty$. In this limit, the oscillator on the (pseudo)sphere results in the conventional circular oscillator

$$
H=2 \pi \bar{\pi}+\frac{\alpha^{2} z \bar{z}}{2}, \quad \omega=d \pi \wedge d z+d \bar{\pi} \wedge d \bar{z},
$$

which possesses the hidden $s u(2)$ symmetry given by the constants of motion

$$
\begin{gathered}
J=i(\pi z-\bar{\pi} \bar{z}), \quad \mathbf{I}=2 \pi^{2}+\alpha^{2} \bar{z}^{2} / 2: \\
\{\overline{\mathbf{I}}, \mathbf{I}\}=4 i \alpha^{2} J, \quad\{\mathbf{I}, J\}=2 i \mathbf{I} .
\end{gathered}
$$

The canonical transformation (14) remains unchanged, the energy level of oscillator converts into the energy level of the Coulomb problem with coupling constant $E / 2$ and the energy $-\alpha^{2} / 2$. The oscillator's constants of motion $J$ and I yield, respectively, the doubled angular momentum and the doubled Runge-Lenz vector

$$
\mathbf{A}=-4 i p J+\frac{E}{2} \frac{\bar{w}}{|w|} .
$$

In the quantum case, the even states of the oscillator yield the conventional Coulomb system, while the odd states yield the Coulomb system in the presence of magnetic vortex generating spin $1 / 2[6]$.

Let us briefly discuss the Bohlin transformation for the oscillator on the (pseudo)sphere interacting with constant magnetic field $B_{0}$. This system can be defined by the following replacement of the symplectic structure (6) and of the rotation generators (7) 


$$
\omega \rightarrow \omega+B_{0} \frac{i 4 R_{0}^{2} d z \wedge d \bar{z}}{(1+\epsilon z \bar{z})^{2}}, \quad J_{i} \rightarrow J_{i}+4 R_{0} B_{0} x_{i},
$$

which preserves the algebra (8) and shifts the initial Hamiltonian (10) on the constant $\left(4 B_{0}\right)^{2}$.

Consequently, the modified system also possesses the hidden symmetry given by (11), (12). The Bohlin transformation (14) of the modified oscillator yields the Coulomb system on the pseudosphere interacting with the magnetic field

$$
B_{C}=\frac{B_{0}}{2 r_{0}}\left(\frac{x_{(C) 3}}{|\mathbf{x}|}-\epsilon\right) .
$$

It is easy to see, that the $2 p$-dimensional oscillator on (pseudo)sphere can be connected to the $(p+$ 1 )-dimensional Coulomb-like systems on pseudosphere in the same manner as in higher dimensions $(p=2,4)$. Indeed, in stereographic coordinates, the oscillator on $2 p$ dimensional (pseudo)sphere is described by the Hamiltonian system given by (6), (10), where the following replacement is performed $(z, \pi) \rightarrow\left(z^{a}, \pi_{a}\right), a=1, \ldots, p$ with the summation over these indices. Consequently, the oscillator's energy surfaces are of the form (13). Further reduction to the $(p+1)$-dimensional Coulomb-like system on pseudosphere repeats the corresponding reduction in the flat case [7, 8].

For example, if $p=2$, we reduce the system under consideration by the Hamiltonian action of the $U(1)$ group given by the generator

$$
J=i(z \pi-\bar{z} \bar{\pi}) .
$$

For this purpose, we have to fix the level surface $J=2 \mathrm{~s}$ and choose the $U(1)$-invariant stereographic coordinates in the form of conventional Kustaanheimo-Stiefel transformation [3] (see also [7])

$$
\mathbf{u}=z \boldsymbol{\sigma} \bar{z}, \quad \mathbf{p}=\frac{z \boldsymbol{\sigma} \pi+\bar{\pi} \boldsymbol{\sigma} \bar{z}}{2(z \bar{z})}
$$

where $\boldsymbol{\sigma}$ are the Pauli matrices.

As a result, the reduced symplectic structure reads

$$
d \mathbf{u} \wedge d \mathbf{p}+s \frac{(\mathbf{u} \times d \mathbf{u}) \wedge d \mathbf{u}}{|\mathbf{u}|^{3}},
$$

while the oscillator's energy surface takes the form

$$
\frac{\left(1-\mathbf{u}^{2}\right)^{2}}{8 r_{0}^{2}}\left(\mathbf{p}^{2}+\frac{s^{2}}{\mathbf{u}^{2}}\right)-\frac{\gamma}{r_{0}} \frac{1+\mathbf{u}^{2}}{2|\mathbf{u}|}=\mathcal{E}_{C},
$$

where $r_{0}, \gamma, \mathcal{E}_{C}$ are defined by the expressions (16).

Interpreting $\mathbf{u}$ as the real stereographic coordinates of three-dimensional pseudosphere

$$
\mathbf{x}=r_{0} \frac{2 \mathbf{u}}{1-\mathbf{u}^{2}}, \quad x_{4}=r_{0} \frac{1+\mathbf{u}^{2}}{1-\mathbf{u}^{2}},
$$

we conclude that (36) defines the energy surface of the pseudospherical analog of a Coulomb-like system proposed in Ref. 14], that describing the interaction of two non-relativistic dyons.
In $p=4$ case, we have to reduce the system by the action of the $S U(2)$ group and choose the $S U(2)$-invariant stereographic coordinates and momenta in the form corresponding to the standard Hurwitz transformation [4] 8 ] which yields a pseudospherical analog of the so-called $S U(2)$-Kepler (or Yang-Coulomb) system [8].

Acknowledgments. The authors are grateful to V. M. Ter-Antonyan for valuable discussions and C. Groshe for drawing their attention to Ref. [5]. A.N. thanks D. Fursaev and C. Sochichiu for useful comments and interest in the work. The work of G.P. is partially supported by RFBR grants $98-01-00330$ and 00-02-81023.

[1] M. Bander, C. Itzykson, Rev. Mod. Phys. 38 (1966), 330. J. Moser, Comm. Pure Appl. Math.23(1970) 609

[2] K. Bohlin, Bull. Astr., 28 (1911), 144 T. Levi-Civita, Opere Mathematiche,2(1906), 411

[3] P. Kustaanheimo, E. Stiefel, J. Reine Angew Math., 218 (1965), 204

[4] A. Hurwitz, Mathematische Werke, Band II, 641 (Birkhäuser, Basel, 1933)

L. S. Davtyan et al, J. Phys. A20 (1987), 6121;

D. Lambert, M. Kibler, J. Phys. A21 (1988), 307

[5] C. Groshe, F. Stainer, Handbook of Feinman path integrals, Springer Tracts in Modern Physics, 145

[6] A. Nersessian, V. M. Ter-Antonyan, M. Tsulaia, Mod. Phys. Lett. A11 (1996), 1605;

A. Nersessian, V. Ter-Antonyan, Phys. Atom. Nucl. 61 (1998) 1756

[7] T. Iwai, Y. Uwano, J. Math. Phys. 27 (1986), 1523 A. Nersessian, V. Ter-Antonyan, Mod. Phys. Lett. A9 (1994), 2431; A10 (1995), 2633

[8] T. Iwai, J. Geom. Phys. 7 (1990), 507; L. G. Mardoyan, A. N. Sissakian, V. M. Ter-Antonyan, Phys. Atom. Nucl. 61 (1998), 1746

[9] E. Schrödinger, Proc. Roy. Irish Soc. 46 (1941) 9; 46 (1941) $183 ; 47$ (1941) 53

[10] P. W. Higgs, J. Phys. A: Math. Gen. 12 (1979) 309 H. I. Leemon, J. Phys. A: Math. Gen. 12 (1979) 489

[11] A. Barut, A. Inomata, G. Junker, J. Phys. A20 (1987), 6271; J. Phys. A23 (1990), 1179 Ya. A. Granovsky, A .S. Zhedanov, I. M. Lutzenko, Teor. Mat. Fiz.91 (1992) 207; 91 (1992) 396;

D. Bonatos, C. Daskaloyanis, K. Kokkatos, Phys. Rev. A50 (1994) 3700;

C. Grosche, G. S. Pogosyan, A. N. Sissakian, Fortschritte der Physik, 43 (6), (1995) 523;

E. G. Kalnins, W. Miller Jr., G. S. Pogosyan, J. Math. Phys. 37 (1996) 6439;38 (1997) 5416

[12] E. G. Kalnins, W. Miller Jr., G. S. Pogosyan, J. Math. Phys.37(2000), 2629

[13] F. Wilczek, Phys. Rev. Lett. 48 (1982), 114

[14] D. Zwanziger, Phys. Rev. 176 (1968), 1480 